\title{
Towards fault-tolerant decision support systems for ship operator guidance
}

\author{
Nielsen, Ulrik Dam; Lajic, Zoran; Jensen, Jørgen Juncher
}

Published in:

Reliability Engineering \& System Safety

Link to article, DOI:

10.1016/j.ress.2012.04.009

Publication date:

2012

Link back to DTU Orbit

\section{Citation (APA):}

Nielsen, U. D., Lajic, Z., \& Jensen, J. J. (2012). Towards fault-tolerant decision support systems for ship operator guidance. Reliability Engineering \& System Safety, 104, 1-14. https://doi.org/10.1016/j.ress.2012.04.009

\section{General rights}

Copyright and moral rights for the publications made accessible in the public portal are retained by the authors and/or other copyright owners and it is a condition of accessing publications that users recognise and abide by the legal requirements associated with these rights.

- Users may download and print one copy of any publication from the public portal for the purpose of private study or research.

- You may not further distribute the material or use it for any profit-making activity or commercial gain

- You may freely distribute the URL identifying the publication in the public portal 


\title{
TOWARDS FAULT-TOLERANT DECISION SUPPORT SYSTEMS FOR SHIP OPERATOR GUIDANCE
}

\author{
Ulrik D. Nielsen ${ }^{a}$, Zoran Lajic ${ }^{b}$ and Jørgen J. Jensen ${ }^{a}$ \\ ${ }^{a}$ Department of Mechanical Engineering, Technical University of Denmark, Kgs. Lyngby, Denmark \\ ${ }^{b}$ A.P. Møller-Marsk, Copenhagen, Denmark
}

\begin{abstract}
Fault detection and isolation are very important elements in the design of faulttolerant decision support systems for ship operator guidance. This study outlines remedies that can be applied for fault diagnosis, when the ship responses are assumed to be linear in the wave excitation. A novel numerical procedure is described for the calculation of residuals using the ship's transfer functions which correlate the wave excitation and the ship responses. As tests, multiplicative faults have artificially been imposed to full-scale motion measurements and it is shown that the developed model is able to detect and isolate all faults.
\end{abstract}

Key words: Fault diagnosis; system models; system reliability; transfer functions; fault-tolerant decision support systems; sea state estimation

\section{INTRODUCTION}

Today, onboard monitoring and decision support systems are installed on many commercial and navy vessels. In the present context, 'decision support' relates to operator guidance for the ship's master with respect to deciding on course and speed to keep wave-induced responses (motions, accelerations, etc.) below an acceptable limit. Typically, several responses are monitored by measurements from a system of sensors that could be as indicated in Figure 1. The decision support system (DSS) is conceptually based on a principle which involves hydrodynamical and mathematical models in combination with information about the sea state (see sub-section 1.2). The overall idea is sketched in Figure 2, where 'Output' is guidance in terms of predicted responses. The predicted responses apply to given operational conditions, including speed and course, and will facilitate an evaluation of risks associated to the considered conditions. In the end, this should lead to future response measurements below acceptable limits.

Decision support systems should be applied only when the quality of associated sensors and software is tested, securing the whole integrated system to be generally well-working, Nielsen et al. [23]. On 


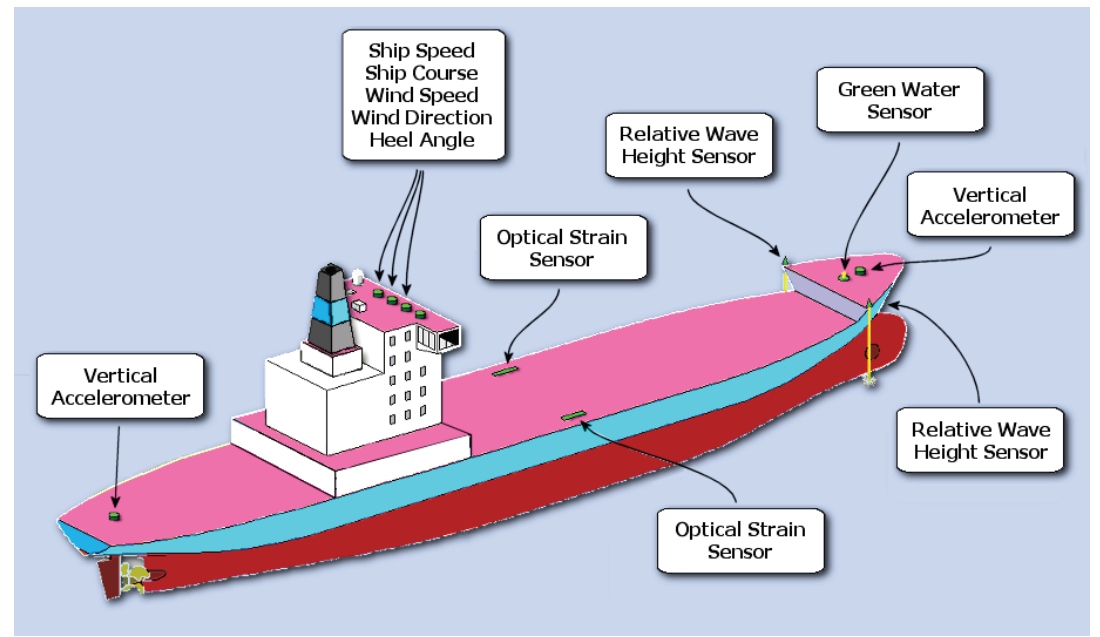

FiguRE 1. Example of sensor arrangement on a ship.

the other hand, it can never be avoided that sensors, or their corresponding signals, at some stage, are likely to be corrupted by faults. It is therefore vital to be able to automatically detect any faults that may occur in a decision support system during operation, so that information/warnings can be issued about unreliable results. As a direct extension to 'fault detection' comes a wish to make a decision support system fault-tolerant. This means that the system has the ability to react on the existence of fault(s) by adjusting its activities to the faulty behaviour of the system. Fault-tolerant decision support systems for ship operator guidance are not standard, and little work has been done so far in the area. However, recently research has been initiated, e.g. Lajic [15], Lajic and Nielsen [17], Lajic et al. [16, 18], and it is foreseen that many improvements in this area will be made in the future.

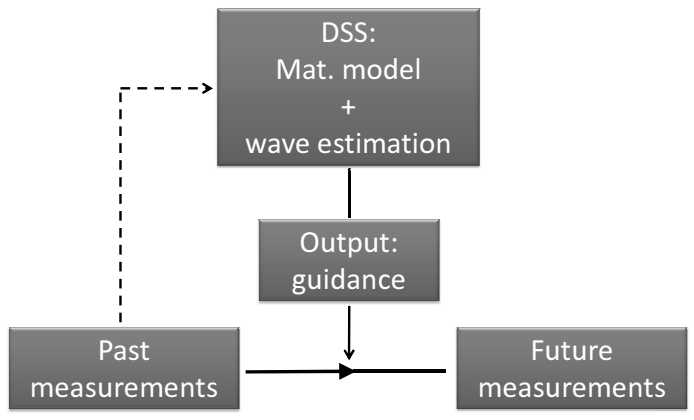

FIGURE 2. Guidance is provided from a mathematical model and statistics of past measurements can be used to supplement shipboard wave estimations in the mathematical model. Nielsen and Jensen [22]. 
The present article summarises important findings made in a recent Ph.D. work, Lajic [15], on faulttolerant monitoring and decision support systems. Specifically, work was carried out to improve the reliability and dependability of decision support systems applied for matters of ship safety. This included the development of algorithms incorporating fault diagnosis techniques and improving the multi-sensor data fusion taking place in most monitoring and decision support systems.

1.1. Literature. Although the introduction of fault diagnosis techniques is new within the scope of decision support systems for ship safety, there is an existing literature on fault-tolerant approaches and algorithms for control of general ship components (engine, propulsion system, rudder, etc.) and particular responses. The present work has its foundation in some of this literature which includes: Three papers by Blanke $[3,4,6]$ in which fault diagnosis and fault-tolerant control approaches are advocated for as means to enhance maritime and navigational safety. In Blanke et al. [7] the reconfiguration possibilities for a ship propulsion system with a main engine and a controllable pitch propeller have been analysed and it has been demonstrated how fault tolerance could be achieved against a critical sensor failure. Similarly, sensor fault-tolerant control for a ship propulsion system is presented in Wu et al. [31], and implementation of the onboard control and monitoring system for unmanned underwater vehicles has been presented in Tiano et al. [30]. Furthermore, applications have been presented for early detection of parametric roll by Galeazzi et al. [10, 11].

1.2. Sea state estimation. A delicate and crucially fundamental part of decision support systems is the part which concerns the onboard estimation of the sea state at the advancing ship's

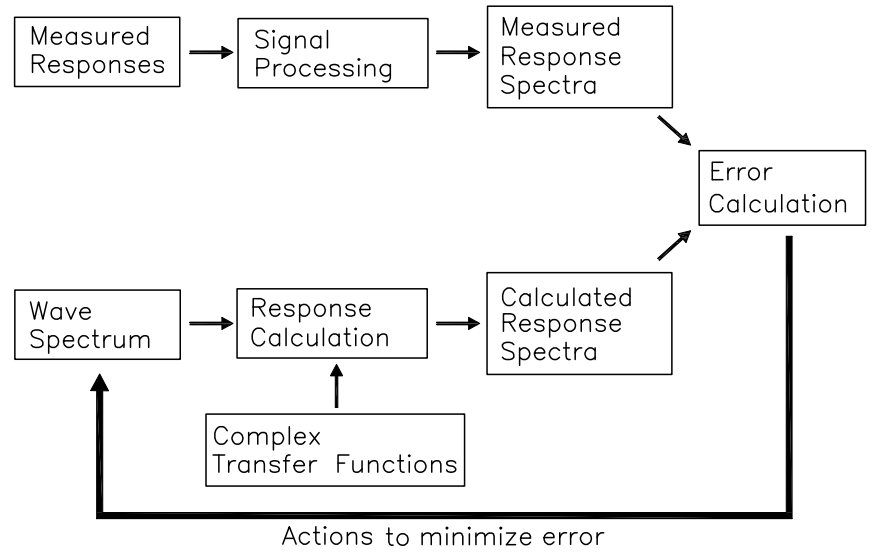

FiguRE 3. The fundamental idea in the estimation of wave spectra based on measured ship responses. 
exact position in the ocean. Thus, the combined use of the estimated sea state and mathematical/hydrodynamical models of the ship's behaviour to waves makes it possible to (statistically) predict about the future responses of the ship expected in, say, a 30 minutes horizon. Basically, two approaches exist for onboard sea state estimation: (1) the wave buoy analogy, which processes measured ship responses to give an estimate of the wave environment, and (2) wave radar systems. The use of satellite data is not yet a feasible approach for onboard wave measurements, although in the future this could change. The present work relies on the wave buoy analogy $[12,20,21,24,27,26,29]$ where the fundamental input is a set of response measurements (motions, accelerations, strains, etc.). In this way, the way buoy analogy utilises onboard response measurements that are often carried out irrespectively on many of today's naval and commercial vessels. Consequently, the wave buoy analogy is a relatively inexpensive estimation concept, since the system development is associated with software only [24]. The wave buoy analogy as such will not be considered herein but a graphical illustration of the procedure is given in Figure 3 [1] and detailed information can be found in the given references.

1.3. Objectives. The overall objective of this work is to improve the reliability and dependability of decision support systems for ship operator guidance with respect to wave-induced responses. In general, the following techniques are suggested to improve the overall reliability and dependability of onboard systems:

- Fault diagnosis - means to detect the presence of faults in the system. Faulty signal(s) should be discarded from the procedure for sea state estimation if it is possible, if not the fault should be estimated. When the sea state estimation is conducted by the wave buoy analogy, e.g. [20, 21], it is sufficient to use three different ship responses and usually the responses of more sensors are available.

- Sensor fusion quality test - means to decide which three ship responses would be the most suitable combination for wave spectrum estimation. The sensor fusion quality test should be applied to each combination of three non-faulty signals.

It is noteworthy that this article deals with the first item - Fault diagnosis - only. This choice is made for matters of space limitations and since the sensor fusion quality test still needs to be elaborated on.

In the original work by Lajic [15] two models for fault diagnosis are discussed: the time domain model based on ship kinematics, and the frequency domain model based on linear spectral analysis. 
By use of linear spectral analysis, it is possible to define equations which correlate different ship responses through transfer functions. With the frequency domain model, all faults can be isolated and their magnitudes can be estimated. On the other hand, using the time domain model independently, all the faults can be detected, but not isolated. This means that additional techniques should be employed, like active fault detection or direct evaluation of Gaussian zero-mean ship responses by the GLR (Generalized Likelihood Ratio) test. In this article, focus will therefore be on the frequency domain model only. It should be noted that the frequency domain model, using transfer functions for calculating the residuals, is a new numerical procedure for fault diagnosis.

1.4. Composition of article. The article has been divided into six main sections. The next section, Section 2, briefly describes the main principles of fault-tolerant onboard decision support systems and the basics of fault diagnosis will be introduced. Moreover, the frequency domain (system) model will be derived. Section 3 introduces system analysis and elaborates on the system model by application of structural analysis (of the system structure). The goal of the section is to derive the residuals, which are the primary target for fault detection and isolation. Reliable change detection algorithm is a fundamental element for fault detection and some basics of change detectors are given in Section 4. In Section 5, full-scale data is considered and it is shown that fault detection and isolation can be achieved by the developed model. Finally, conclusions are made in Section 6 which also recommends future work.

\section{FAULT-TOLERANT MONITORING AND DECISION SUPPORT SYSTEMS}

Although the following section is written with a focus on monitoring and decision support systems the terminology is relaxed, so that only systems will be mentioned (except for the last sub-section). In this regards, one of several definitions of a 'system' should be kept in mind, Miller [19]: "A system is a set of interacting units with relationship among them". The section introduces some standard terms and general principles for fault diagnosis, which relies highly on Blanke et al. [8]. At the end of the section, a documentation will be given of the specific system model which will be analysed later with respect to full-scale measurements.

2.1. System behaviour. A fault is something that changes the system behaviour, so that the system no longer serves its purpose. The fault is the primary cause of system performance degradation or even loss of the system function. Therefore, it is very important to find the fault(s) as quickly as possible and to make decisions that stop the propagation of their effects. The aim of 


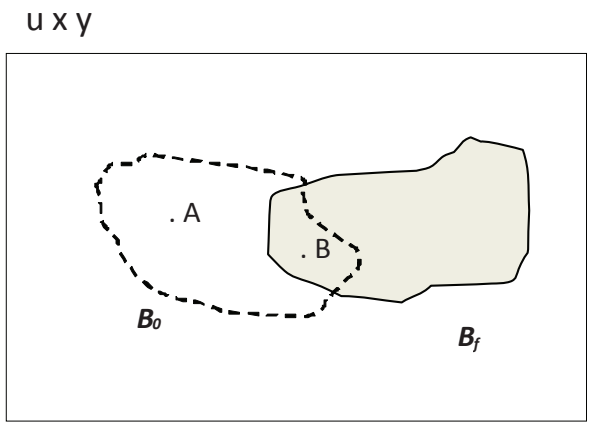

Figure 4. System beaviour; $\boldsymbol{B}_{0}$ is a faultless case whereas $\boldsymbol{B}_{f}$ is a faulty case.

these measures is to make the system fault-tolerant meaning that the system is still functioning after the appearance of a fault. A fault-tolerant system has the ability to react on the existence of the fault by adjusting its activities to the faulty behaviour of the system. The procedure for making a system fault-tolerant consists of two steps, cf. Blanke et al. [8]:

(1) Fault diagnosis: The existence of faults has to be detected and the faults have to be identified.

(2) Re-design: The system has to be adapted to the faulty situation so that the overall system continues to satisfy its goal.

A system with an input $u(t)$ and an output $y(t)$ both depending on time $t$ is now considered. The pair $(u, y)$ is called input/output (I/O) pair, and the set of all possible pairs that may occur for a given system defines the behaviour $\boldsymbol{B}$. The behaviour $\boldsymbol{B}$ can be defined as a subset of the space $u \times y$ of all possible combinations of I/O signals. In Figure 4 a graphical interpretation of the system behaviour is given. From the figure two particular system behaviours $\boldsymbol{B}_{0}$ (faultless) and $\boldsymbol{B}_{f}$ (faulty) are considered. The point A represents an I/O pair that may occur for the faultless system, while the point B represents a pair which is inconsistent with the system dynamics. This means that the point $\mathrm{B}$ corresponds to an I/O pair influenced by a fault.

In general, any fault changes the system behaviour. Thus, the consequence of a fault is that the system behaviour moves from the set $\boldsymbol{B}_{0}$ towards the set $\boldsymbol{B}_{f}$. In the case of a common input $u$, the two systems - faultless and faulty - will give different outputs $y_{A}$ and $y_{B}$, respectively. The change in the system behaviour makes the detection and isolation of a fault possible. However, the measurement information $(u, y)$ alone is not sufficient, but a dynamical model, which describes the nominal system behaviour, is necessary. 
The system model describes the behaviour of the faultless and the faulty system which means that the model restricts the possible I/O pairs to those that appear in the behaviour $\boldsymbol{B}_{0}$ or $\boldsymbol{B}_{f}$. In other words, the model is a set of constraints on the signals $u$ and $y$ in the system. In fault diagnosis both the input $u$ and the output $y$ will be known and it is checked whether the I/O pair belongs to the specific behaviour.

2.2. The diagnostic principle. If a specific I/O pair $(U, Y)$ is represented by a point A, cf. Figure 4, then for a faultless system (and a correct system model) A lies in the set $\boldsymbol{B}_{0}$. On the contrary, a different output $\widehat{Y}$ is generated from $U$ if the system is faulty. The particular fault is said to be detectable if the I/O pair $(U, \widehat{Y})$ lies outside $\boldsymbol{B}_{0}$. However, if the produced I/O pair corresponds to point B, cf. Figure 4, the faulty system leads to no inconsistency and the fault is not detected. This consideration leads to the foundation of the diagnostic principle which is concerned with testing whether or not any measurement $(U, Y)$ is consistent with the system behaviour. Thus, any I/O pair should be checked with respect to the nominal system behaviour and, in case of a fault, the fault is detected if $(U, Y) \notin \boldsymbol{B}_{0}$. However, if the I/O pair lies in $\boldsymbol{B}_{f}$ for a system subject to a fault $f$, the fault may occur. Hence, $f$ is denoted a fault candidate. The diagnostic result is usually a set $\mathcal{F}_{c} \subseteq \mathcal{F}$ of fault candidates.

In summary, the diagnostic principle is based on the following test:

Consistency-based diagnosis - For given models that describe the behaviour $\boldsymbol{B}_{f}$ of the system subject to the faults $f \in \mathcal{F}$, test whether the I/O pair $(U, Y)$ satisfies the relation

$$
(\mathrm{U}, \mathrm{Y}) \in \boldsymbol{B}_{f}
$$

From the test, two outcomes are considered:

- Fault detection - If the I/O pair is inconsistent with the behaviour $\boldsymbol{B}_{0}$ of the faultless system, $(U, Y) \notin \boldsymbol{B}_{0}$, then a fault is know to have occurred.

- Fault isolation and identification - If the input/output pair is consistent with the behaviour $\boldsymbol{B}_{f}$, that is, $(U, Y) \in \boldsymbol{B}_{f}$, then the fault may have occurred.

On the basis of the diagnostic principle and its consequences it should be noted that fault detection is possible without any information about the behaviour of the faulty system. Fault detection algorithms use only a model of the nominal system. It is sufficient to identify deviations of the current 
system behaviour from the nominal behaviour. On the other hand, fault isolation and identification are not possible without information about the faulty system. Therefore, corresponding fault models have to be known.

2.3. Diagnostic algorithms. In fault diagnosis, the sensor measurement $Y$ is compared with the analytically computed value $\widehat{Y}$. Therefore, the consistency of the system with the model can be tested at every time $t$ by forming the residual

$$
r(t)=y(t)-\widehat{y}(t)
$$

In the faultless case, the residual is close to zero. In general, it is not exactly zero due to measurement noise and model uncertainties. If any fault exists, the residual has some specific, non-vanishing value.

As pointed out by Blanke et al. [8], diagnostic algorithms for continuous-variable systems generally consist of two components, see also Figure 5:

- Residual generation: The system model and the I/O pair are used to determine residuals which describe the degree of consistency between the system and the model behaviour.

- Residual evaluation: The residual is evaluated in order to detect, isolate and identify faults.

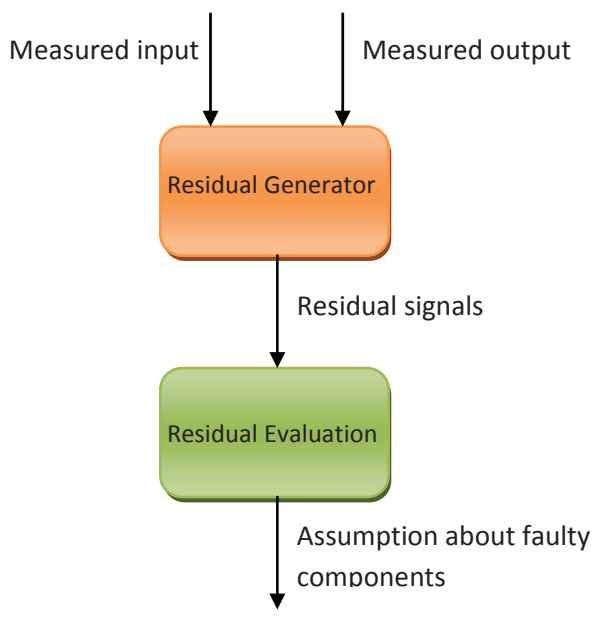

FIGURE 5. Residual generation and evaluation are necessary components of diagnostic algorithms.

2.4. Re-design. The second step towards a fault-tolerant system involves re-design, which considers the problem of changing the system structure after a fault has occurred. The aim is to make 
the system able to serve its purpose after the occurrence of a fault. Two principal ways of re-design have to be distinguished:

- Fault accommodation (off-line solution): Fault accommodation means to adapt the parameters to the dynamical properties of the faulty system. The input and output of the system remain the same as for the faultless case. Fault accommodation is based on predesigned systems, each of which has been selected off-line for a specific fault. This method is fast and can meet strong real-time constraints. However, re-design has to be made for all possible faults before the system is operative.

- Reconfiguration (on-line solution): If fault accommodation is impossible, the complete system has to be reconfigured. In this case a new system configuration is obtained, where alternative input and output signals are used. Reconfiguration is necessary in case of sensor failure.

Although re-design is an important part of a fault-tolerant decision support system this part will not be considered in the present paper.

2.5. The particular system model. In the preceding, basic terms of fault diagnosis were introduced, where the importance of system models has been reflected. In this sub-section, the particular system model, which will be analysed with data later, is documented. In Lajic [15], system models have been derived both for the time domain and the frequency domain. The focus herein is, however, on the frequency domain model only.

The responses of a ship in an irregular (long-crested) seaway can be deduced by the characteristic wave energy spectrum $S_{\zeta}(\omega)$ and the ship's transfer functions $\Phi(\omega)$, where $\omega$ is the wave frequency. In this way, the ship responses can be described by response spectra [28], where the spectral densities are equal to the product of the spectral density of the waves and the square of the transfer functions. Therefore, in the case of long-crested waves, it follows that the response spectra of, say, heave, $z$, pitch, $\theta$, roll, $\varphi$ and vertical acceleration, $a$, amidships are:

$$
\begin{array}{ll}
c_{1}: & S_{z}\left(\omega_{e}\right)=\Phi_{z}^{2}\left(\omega_{e}\right) S_{\zeta}\left(\omega_{e}\right) \\
c_{2}: & S_{\theta}\left(\omega_{e}\right)=\Phi_{\theta}^{2}\left(\omega_{e}\right) S_{\zeta}\left(\omega_{e}\right) \\
c_{3}: & S_{\varphi}\left(\omega_{e}\right)=\Phi_{\varphi}^{2}\left(\omega_{e}\right) S_{\zeta}\left(\omega_{e}\right) \\
c_{4}: & S_{a}\left(\omega_{e}\right)=\Phi_{a}^{2}\left(\omega_{e}\right) S_{\zeta}\left(\omega_{e}\right)
\end{array}
$$

which will be the constraints, $c_{i}$, of the system model. 
In Eqs. (1)-(4), the encounter frequency $\omega_{e}$ has been introduced, since the response spectra are derived from sensor measurements onboard the ship. The relationship between the encountered frequency and the true wave frequency is governed by the Doppler shift

$$
\omega_{e}=\omega-\omega^{2} A \quad, \quad A=\frac{U}{g} \cos \chi
$$

for the relative wave heading $\chi$ and speed $U ; g$ is the acceleration of gravity. The transfer functions of a ship are measures of the ship's seakeeping performance. Typically, the transfer functions are obtained by linear frequency domain strip theory, three-dimensional panel codes, or by measurements.

In addition to the constraints, $c_{1}, \ldots c_{4}$, the following transforms, $t_{i}$, and measurements, $m_{i}$, are introduced:

$$
\begin{aligned}
t_{1}: & S_{z}\left(\omega_{e}\right) & =\mathfrak{F}(z) \\
t_{2}: & S_{\theta}\left(\omega_{e}\right) & =\mathfrak{F}(\theta) \\
t_{3}: & S_{\varphi}\left(\omega_{e}\right) & =\mathfrak{F}(\varphi) \\
t_{4}: & S_{a}\left(\omega_{e}\right) & =\mathfrak{F}(a) \\
m_{1}: & y_{1} & =z \\
m_{2}: & y_{2} & =\theta \\
m_{3}: & y_{3} & =\varphi \\
m_{4}: & y_{4} & =a
\end{aligned}
$$

In the present case, the symbol $\mathfrak{F}(\quad)$ denotes a transform operator which transforms from time domain to the frequency domain. It could for example be the Fast Fourier transform (FFT).

For the studied system model - dealing generally with wave-ship interactions - it seems relevant to make a couple of notes about the implication of (particularly) rough sea conditions. Firstly, this paper is intended to illustrate initial ideas developed for fault-tolerant decision support systems and it is therefore considered to be of less importance how accurately the hydrodynamic behaviour of a ship is calculated. For this reason, all data and results studied later are derived on the basis of transfer functions (obtained by the 3D panel code Wasim [9]) without going into details about accuracy. However, it is clear that for rough wave conditions, the use of transfer functions can generally lead to unreliable results due to the inherent linear assumption, which is often violated in rough sea conditions. On the other hand, it should be stressed that the suggested system model 
- based on frequency domain theory, facilitating the use of transfer functions - can be extended to include also time domain theory, see [15], without introducing a linear assumption between the wave-ship interactions. As a second note, it is worth to point out that during rough sea conditions, fault detection is particularly useful, since any system sensor may observe continuous structural vibrations. Thus, fault detection (and tolerance) is fundamental to secure a system's performance also in the event of degrading sensors due to unintended vibrations. However, the influence of the structural vibrations could/should be limited by a proper installation of the sensors.

\section{System AnALYSis}

3.1. Introduction. In order to have a fault-tolerant onboard decision support system, fault diagnosis is a crucial element. Therefore, already at an early design stage it is important to secure that it is possible to detect and isolate faults. The usual way to do this is to apply structural analysis (which should not be confused with the twin term within the fields of mechanical and civil engineering). In this section the structure of the studied (dynamical) system will be investigated. By applying structural analysis, it is possible to obtain answers regarding fault diagnosis without detailed calculations, e.g. the ability to detect the fault or recover after the fault. Structural analysis can be performed from the early design stages where detailed modelling has not yet been made. For sensor fault detection, there is a need to find physical relations between measured values. There is no need to express those physical relations explicitly. Rather, the structure of the constraints, that is the existence of relations between variables and parameters, is considered only.

Example. Basically, structural analysis investigates the links which exists between variables and parameters, independently on the form of the underlying equations [8]. Often, structural analysis is therefore performed by use of graph theory, where a bi-partite graph and an associated incidence matrix represents a "qualitative, very low-level, easy to obtain, model of the system behaviour", Blanke et al. [8]. This can be illustrated by a small-scale example, where a system described by the following equation is studied:

$$
P_{B}=2 \pi Q n
$$

$P_{B}$ is the brake power of an engine, $Q$ is the brake torque, and $n$ is the revolutions per second. The equation can be transformed into the following form:

$$
c_{1}: \quad P_{B}-2 \pi Q n=0
$$


which means that the constraint $c_{1}$ relates three variables $\left(P_{B}, Q, n\right)$. Furthermore, the brake power is measured by a sensor whose output is $y_{1}$, and the revolutions per second $n$ by a sensor whose output is $y_{2}$. This means that the previous system obeys the expressions:

$$
\begin{array}{cl}
c_{1}: & P_{B}-2 \pi Q n=0 \\
m_{1}: & y_{1}-P_{B}=0 \\
m_{2}: & y_{2}-n=0
\end{array}
$$

The known variables are $y_{1}$ and $y_{2}$ only, because they are the measured sensor outputs. All other variables are unknown. The relations in the system can be summarised as follows:

$c_{1}$ relates $P_{B}, Q$ and $n$

$m_{1}$ relates $P_{B}$ and $y_{1}$

$m_{2}$ relates $n$ and $y_{2}$

In the early design phase of a fault-tolerant system, these relations are the only concern. The structure of the system is presented at the right-hand side in Figure 6 (the incidence matrix), where rows represent the set of constraints and columns the set of variables. If a particular variable is a member of a particular constraint, '1' will be written in the cell, and if not the cell will be empty. Another way of representing the relations in the system is to use the graph shown at the left-hand side in Figure 6. It is worth noting that both the table and the graph give the same information about the system.

By inspection of the incidence matrix (or the bi-partite), it can be concluded that all the unknown variables in the system can be computed, since $P_{B}$ can be computed from $y_{1}$ by using the measurement equation $m_{1}$ (this is denoted by the bold red 1 ), $n$ can be computed from $y_{2}$ by using the measurement equation $m_{2}$ and therefore $Q$ can be computed from the constraint $c_{1}$. However, there is no need to calculate all the variables. The important thing is to see if it is possible to compute all variables and if there are some equations which have not been used for computing variables.

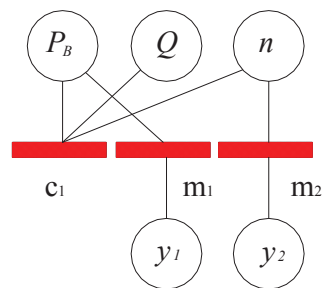

\begin{tabular}{|l||r|r|r|r|r|}
\hline$\nearrow$ & $y_{1}$ & $y_{2}$ & $P_{B}$ & $Q$ & $n$ \\
\hline$c_{1}$ & & & 1 & $\mathbf{1}$ & 1 \\
\hline$m_{1}$ & 1 & & 1 & & \\
\hline$m_{2}$ & & 1 & & & $\mathbf{1}$ \\
\hline
\end{tabular}

FIGURE 6. System structure; bi-partite graph (left) and incidence matrix (right). 
The latter equations will be used for extracting the residuals. In the presence of a fault, the system components no longer satisfy the equations which are defined for their normal behaviour. Therefore, structural analysis can give the possibility of detecting faults. A fault-detection procedure will be presented in Section 5, where the residuals will be evaluated.

Onboard systems. Most onboard systems are very complex and therefore there is a need for having a tool to cope with the complexity of the systems. Structural analysis based on graph theory is a unique design methodology that can cope with the diagnosis design for systems of high complexity and also be used for analysis of the cases of cascaded or multiple faults, cf. Blanke [5]. From a bi-partite graph, it is possible to obtain information about the relations between variables, but it is not possible to obtain information about the appearance of the constraints. If two systems have the same bi-partite graph, those systems are structurally equivalent. It is noteworthy that systems, which have the same structure but only differ by the values of their parameters, are still structurally equivalent. If a fault is considered as a violation of a constraint, it is obvious that for fault diagnosis, the structure is of essential concern. The values of system parameters do not influence the ability to detect or isolate a fault in structural analysis, but parameters can have values such that structural detectability does not imply analytical detectability.

In structural analysis, it is convenient to separate the set of system variables into unknown $(X)$ and known $(K)$ sets. In the case of onboard decision support systems, known variables are measured ship responses, e.g. pitch, heave, roll motion, etc. On the other hand, unknown variables are not measured but should instead be computed. In the case of onboard systems, unknown variables include for example the wave spectrum. The wave spectrum is not measured directly; instead it can be calculated/estimated by a procedure that has as input a set of measured ship responses. Like the system variables, the constraints can also be divided into the set $C_{X}$ (unknown) and $C_{K}$ (known). The set $C_{K}$ contains constraints which relate known variables only, and the set $C_{X}$ contains constraints which relate at least one unknown variable. In order to extract the residuals available for fault diagnosis, structural analysis of the constraints and the unknown variables may be applied. While there are several ways to deduct analytical redundancy relations, structural analysis is particularly simple; the details are explained in Lajic [15].

3.2. The frequency domain model. In the following, the frequency domain model of an onboard decision support system is considered. The constraints of the model were derived previously (Eqs. 
1 to 4) but can be rewritten in a form which is more convenient for the system analysis:

$$
\begin{array}{ll}
c_{1}: & \frac{S_{z}\left(\omega_{e}\right)}{\Phi_{z}^{2}\left(\omega_{e}\right)}=S_{\zeta}\left(\omega_{e}\right) \\
c_{2}: & \frac{S_{\theta}\left(\omega_{e}\right)}{\Phi_{\theta}^{2}\left(\omega_{e}\right)}=S_{\zeta}\left(\omega_{e}\right) \\
c_{3}: & \frac{S_{\varphi}\left(\omega_{e}\right)}{\Phi_{\varphi}^{2}\left(\omega_{e}\right)}=S_{\zeta}\left(\omega_{e}\right) \\
c_{4}: & \frac{S_{a}\left(\omega_{e}\right)}{\Phi_{a}^{2}\left(\omega_{e}\right)}=S_{\zeta}\left(\omega_{e}\right)
\end{array}
$$

The transforms, $t_{1}, \ldots, t_{4}$, and measurements, $m_{1}, \ldots, m_{4}$, are given by Eqs. (6)-(9) and Eqs. (10)-(13), respectively.

A bi-partite graph of the system is shown in Figure 7. The system variables are represented by circles and the system constraints by bars. The graph is based on the system equations and it provides an overview of the system. Using the graph, it is possible to see which constraints describe a particular variable. It is interesting to note that the variable $S_{\zeta}$ (the wave energy spectrum) is connected with four constraints $c_{1}, c_{2}, c_{3}, c_{4}$. In the following, it will be shown that $S_{\zeta}$ is not a member of any residual and it will not be calculated (directly). The residuals should contain only known variables, i.e. sensor measurements. The wave spectrum $S_{\zeta}$ will be matched from the constraint $c_{1}$. For fault diagnosis, only the residuals are of interest. The set of variables in the system is separated into the sets $K$ (known) and $X$ (unknown):

$$
K=\left\{y_{1}, y_{2}, y_{3}, y_{4}\right\}
$$

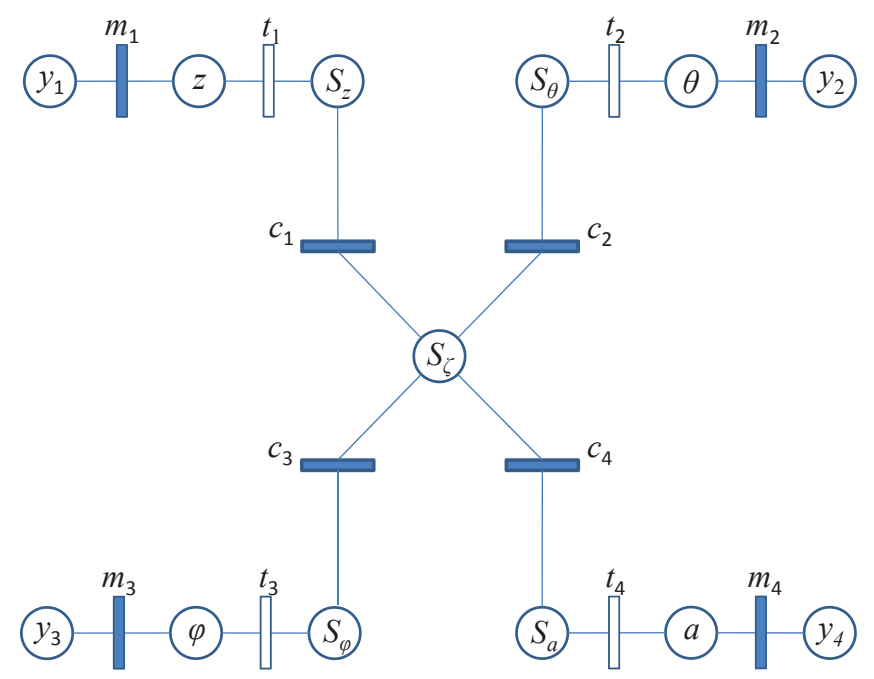

FigURE 7. Bi-partite graph of an onboard system. 


$$
X=\left\{z, \theta, \varphi, a, S_{z}, S_{\theta}, S_{\varphi}, S_{a}, S_{\zeta}\right\}
$$

The constraints depend on the transfer functions of the ship. However, the transfer functions are known functions, since they can be calculated in advance, and they will therefore be considered as system parameters.

The constraints are matched to the unknown variables, i.e. the ship responses, as follows:

$$
\begin{aligned}
& m_{1}\left(y_{1}\right) \rightarrow z \\
& m_{2}\left(y_{2}\right) \rightarrow \theta \\
& m_{3}\left(y_{3}\right) \rightarrow \varphi \\
& m_{4}\left(y_{4}\right) \rightarrow a
\end{aligned}
$$

where the standard notation, indicated by the arrow $(\rightarrow)$, is used to saying that the variable on the right-hand side is obtained (matched) from a given measurement $m_{i}$ that depends on a specific variable.

Previously, the response spectra (of the ship responses) have been matched from the transforms $t_{1} \ldots t_{4}$, cf. Eqs. (6)-(9). On the other hand, the ship responses have already been matched from the measurement equations which means that the following expressions can be derived:

$$
\begin{aligned}
t_{1}(z) & =t_{1}\left(m_{1}\left(y_{1}\right)\right) \rightarrow S_{z} \\
t_{2}(\theta) & =t_{2}\left(m_{2}\left(y_{2}\right)\right) \rightarrow S_{\theta} \\
t_{3}(\varphi) & =t_{3}\left(m_{3}\left(y_{3}\right)\right) \rightarrow S_{\varphi} \\
t_{4}(a) & =t_{4}\left(m_{2}\left(y_{4}\right)\right) \rightarrow S_{a} \\
c_{1}\left(S_{z}\right) & =c_{1}\left(t_{1}\left(m_{1}\left(y_{1}\right)\right)\right) \rightarrow S_{\zeta}
\end{aligned}
$$

Thus, it is possible to attain a complete matching of the unknown variables. However, three constraints remain unmatched. Each of these forms the basis of a residual generator that can check the consistency of the constraints. The residual $r_{1}$ is given by

$$
r_{1}: \quad c_{2}\left[S_{\zeta}, S_{\theta}\right] \rightarrow 0,
$$

and after substitution of the unknown variables, obtained by backtracking through the matching:

$$
r_{1}: \quad c_{2}\left[c_{1}\left(t_{1}\left(m_{1}\left(y_{1}\right)\right)\right), t_{2}\left(m_{2}\left(y_{2}\right)\right)\right] \rightarrow 0
$$


TABLE 1. Incidence matrix.

\begin{tabular}{|l||c|c|c|c|c|c|c|c|c|c|c|c|}
\hline$\nearrow$ & $c_{1}$ & $c_{2}$ & $c_{3}$ & $c_{4}$ & $t_{1}$ & $t_{2}$ & $t_{3}$ & $t_{4}$ & $m_{1}$ & $m_{2}$ & $m_{3}$ & $m_{4}$ \\
\hline$y_{1}$ & 1 & 1 & 1 & 1 & 1 & & & & 1 & & & \\
\hline$y_{2}$ & & 1 & & & & 1 & & & & 1 & & \\
\hline$y_{3}$ & & & 1 & & & & 1 & & & & 1 & \\
\hline$y_{4}$ & & & & 1 & & & & 1 & & & & 1 \\
\hline$z$ & 1 & 1 & 1 & 1 & 1 & & & & 1 & & & \\
\hline$\theta$ & & 1 & & & & 1 & & & & 1 & & \\
\hline$\varphi$ & & & 1 & & & & 1 & & & & 1 & \\
\hline$a$ & & & & 1 & & & & 1 & & & & 1 \\
\hline$S_{z}$ & 1 & 1 & 1 & 1 & $\mathbf{1}$ & & & & & & & \\
\hline$S_{\theta}$ & & 1 & & & & $\mathbf{1}$ & & & & & & \\
\hline$S_{\varphi}$ & & & 1 & & & & $\mathbf{1}$ & & & & & \\
\hline$S_{a}$ & & & & 1 & & & & $\mathbf{1}$ & & & & \\
\hline$S_{\zeta}$ & 1 & 1 & 1 & 1 & & & & & & & & \\
\hline & & $\rightarrow 0$ & $\rightarrow 0$ & $\rightarrow 0$ & & & & & & & \\
\hline
\end{tabular}

The residual $r_{2}$ is given by

$$
r_{2}: \quad c_{3}\left[S_{\zeta}, S_{\varphi}\right] \rightarrow 0
$$

and after substitution

$$
r_{2}: \quad c_{3}\left[c_{1}\left(t_{1}\left(m_{1}\left(y_{1}\right)\right)\right), t_{3}\left(m_{3}\left(y_{3}\right)\right)\right] \rightarrow 0
$$

Finally, the residual $r_{3}$ follows from

$$
\begin{array}{ll}
r_{3}: & c_{4}\left[S_{\zeta}, S_{a}\right] \rightarrow 0, \text { or } \\
r_{3}: & c_{4}\left[c_{1}\left(t_{1}\left(m_{1}\left(y_{1}\right)\right)\right), t_{4}\left(m_{4}\left(y_{4}\right)\right)\right] \rightarrow 0
\end{array}
$$

The incidence matrix and the matching are shown in Table 1. Unmatched constraints are denoted by " $\rightarrow 0 "$. It is easily noted that three constraints are unmatched and that they are the basis of the residual generation. The oriented graph of the system is shown in the upper part of Figure 8 . The output of the unmatched constraints is labelled zero and they are the (three) system residuals. From the oriented graph, it is noted that in order to match $S_{\zeta}, z$ should first be matched from the known variable $y_{1}$ and the measurement equation $m_{1} ; S_{z}$ from the transform $t_{1}$ and, finally, $S_{\zeta}$ 


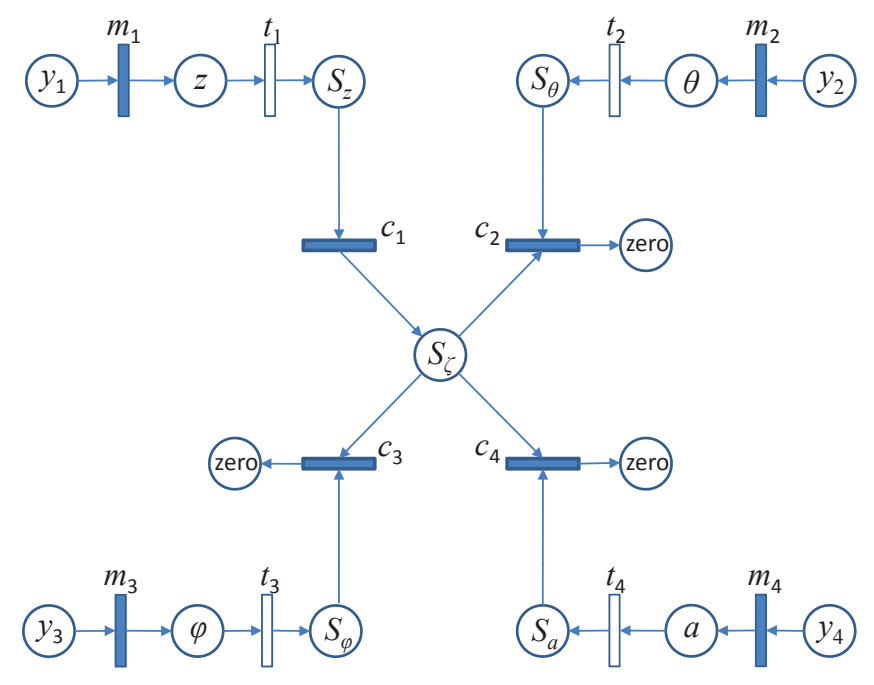

\begin{tabular}{|c||c|c|c|c|c|c|c|c|}
\hline$\nearrow$ & $c_{1}$ & $c_{2}$ & $c_{3}$ & $c_{4}$ & $m_{1}$ & $m_{2}$ & $m_{3}$ & $m_{4}$ \\
\hline$r_{1}$ & 1 & 1 & & & 1 & 1 & & \\
\hline$r_{2}$ & 1 & & 1 & & 1 & & 1 & \\
\hline$r_{3}$ & 1 & & & 1 & 1 & & & 1 \\
\hline & $d$ & $d$ & $d$ & $d$ & $d$ & $d$ & $d$ & $d$ \\
\hline
\end{tabular}

FiguRE 8. Oriented graph (top), in which the arrows indicate the order of matching, and dependency matrix (bottom) of the residuals.

from the constraint $c_{1}$. The dependency matrix of the three residuals is shown in the lower part of Figure 8. It should be noted that transforms $t_{1} \ldots t_{4}$ are omitted from the dependency matrix, because these transforms are based on a mathematical definition and it cannot fail.

The residuals can be expressed in analytical form as

$$
\begin{array}{ll}
r_{1}: & \frac{\left\{\mathfrak{F}\left(y_{1}\right)\right\}\left(\omega_{e}\right)}{\Phi_{z}^{2}\left(\omega_{e}\right)}-\frac{\left\{\mathfrak{F}\left(y_{2}\right)\right\}\left(\omega_{e}\right)}{\Phi_{\theta}^{2}\left(\omega_{e}\right)}=0 \\
r_{2}: & \frac{\left\{\mathfrak{F}\left(y_{1}\right)\right\}\left(\omega_{e}\right)}{\Phi_{z}^{2}\left(\omega_{e}\right)}-\frac{\left\{\mathfrak{F}\left(y_{3}\right)\right\}\left(\omega_{e}\right)}{\Phi_{\varphi}^{2}\left(\omega_{e}\right)}=0 \\
r_{3}: & \frac{\left\{\mathfrak{F}\left(y_{1}\right)\right\}\left(\omega_{e}\right)}{\Phi_{z}^{2}\left(\omega_{e}\right)}-\frac{\left\{\mathfrak{F}\left(y_{4}\right)\right\}\left(\omega_{e}\right)}{\Phi_{a}^{2}\left(\omega_{e}\right)}=0
\end{array}
$$


TABLE 2. Dependency matrix.

\begin{tabular}{|l||c|c|c|c|c|c|c|c|}
\hline$\nearrow$ & $c_{1}$ & $c_{2}$ & $c_{3}$ & $c_{4}$ & $m_{1}$ & $m_{2}$ & $m_{3}$ & $m_{4}$ \\
\hline$r_{4}$ & & 1 & 1 & & & 1 & & \\
\hline$r_{5}$ & & 1 & & 1 & & & 1 & \\
\hline$r_{6}$ & & & 1 & 1 & & & & 1 \\
\hline & & $i$ & $i$ & $i$ & & $i$ & $i$ & $i$ \\
\hline
\end{tabular}

Isolability can be enhanced, however, by creating residuals that are derived from the original three. A combination of these residuals could also be used as residual generators and, by linear combination, the following holds,

$$
\begin{array}{ll}
r_{4}: & \frac{\left\{\mathfrak{F}\left(y_{2}\right)\right\}\left(\omega_{e}\right)}{\Phi_{\theta}^{2}\left(\omega_{e}\right)}-\frac{\left\{\mathfrak{F}\left(y_{3}\right)\right\}\left(\omega_{e}\right)}{\Phi_{\varphi}^{2}\left(\omega_{e}\right)}=0 \\
r_{5}: & \frac{\left\{\mathfrak{F}\left(y_{2}\right)\right\}\left(\omega_{e}\right)}{\Phi_{\theta}^{2}\left(\omega_{e}\right)}-\frac{\left\{\mathfrak{F}\left(y_{4}\right)\right\}\left(\omega_{e}\right)}{\Phi_{a}^{2}\left(\omega_{e}\right)}=0 \\
r_{6}: & \frac{\left\{\mathfrak{F}\left(y_{3}\right)\right\}\left(\omega_{e}\right)}{\Phi_{\varphi}^{2}\left(\omega_{e}\right)}-\frac{\left\{\mathfrak{F}\left(y_{4}\right)\right\}\left(\omega_{e}\right)}{\Phi_{a}^{2}\left(\omega_{e}\right)}=0
\end{array}
$$

The dependency matrix for the modified residuals is shown in Table 2. From the table, the following information about the variables can be obtained by combining residuals $r_{1}$ to $r_{6}$ in the analysis:

Detectable: $c_{1}, m_{1}$

Undetectable: none

Isolable: $c_{2}, c_{3}, c_{4}, m_{2}, m_{3}, m_{4}$

In decision support systems, it is the statistical values of to-be-expected future responses that are of concern. The statistical value could for example be the variance which can be derived from the 0th-order moment defined by

$$
m_{0}=\int_{0}^{\infty} S_{R}(\omega) d \omega
$$

where $S_{R}(\omega)$ is the response spectrum (of any considered response). An analogy can be made to the residuals and therefore it is sufficient to consider integrated versions of Eqs. (30)-(35). Integration over the entire frequency range $\left[\omega_{e, \min }, \omega_{e, \max }\right]$ leads to the following residuals which will be used 
as the final versions for fault detection:

$$
\begin{aligned}
& r_{1}=\int\left[\frac{\left\{\mathfrak{F}\left(y_{1}\right)\right\}\left(\omega_{e}\right)}{\Phi_{z}^{2}\left(\omega_{e}\right)}-\frac{\left\{\mathfrak{F}\left(y_{2}\right)\right\}\left(\omega_{e}\right)}{\Phi_{\theta}^{2}\left(\omega_{e}\right)}\right] d \omega_{e} \\
& r_{2}=\int\left[\frac{\left\{\mathfrak{F}\left(y_{1}\right)\right\}\left(\omega_{e}\right)}{\Phi_{z}^{2}\left(\omega_{e}\right)}-\frac{\left\{\mathfrak{F}\left(y_{3}\right)\right\}\left(\omega_{e}\right)}{\Phi_{\varphi}^{2}\left(\omega_{e}\right)}\right] d \omega_{e} \\
& r_{3}=\int\left[\frac{\left\{\mathfrak{F}\left(y_{1}\right)\right\}\left(\omega_{e}\right)}{\Phi_{z}^{2}\left(\omega_{e}\right)}-\frac{\left\{\mathfrak{F}\left(y_{4}\right)\right\}\left(\omega_{e}\right)}{\Phi_{a}^{2}\left(\omega_{e}\right)}\right] d \omega_{e}
\end{aligned}
$$

And, the auxiliary residuals are,

$$
\begin{aligned}
& r_{4}=\int\left[\frac{\left\{\mathfrak{F}\left(y_{2}\right)\right\}\left(\omega_{e}\right)}{\Phi_{\theta}^{2}\left(\omega_{e}\right)}-\frac{\left\{\mathfrak{F}\left(y_{3}\right)\right\}\left(\omega_{e}\right)}{\Phi_{\varphi}^{2}\left(\omega_{e}\right)}\right] d \omega_{e} \\
& r_{5}=\int\left[\frac{\left\{\mathfrak{F}\left(y_{2}\right)\right\}\left(\omega_{e}\right)}{\Phi_{\theta}^{2}\left(\omega_{e}\right)}-\frac{\left\{\mathfrak{F}\left(y_{4}\right)\right\}\left(\omega_{e}\right)}{\Phi_{a}^{2}\left(\omega_{e}\right)}\right] d \omega_{e} \\
& r_{6}=\int\left[\frac{\left\{\mathfrak{F}\left(y_{3}\right)\right\}\left(\omega_{e}\right)}{\Phi_{\varphi}^{2}\left(\omega_{e}\right)}-\frac{\left\{\mathfrak{F}\left(y_{4}\right)\right\}\left(\omega_{e}\right)}{\Phi_{a}^{2}\left(\omega_{e}\right)}\right] d \omega_{e}
\end{aligned}
$$

\section{Change Detection Algorithms}

4.1. Introduction. In order to recognise the occurrence of a fault, a tool must be provided for real-time fault detection. This can be achieved by applying a detector as part of the residual evaluation. Thus, the detector will inform in real-time to the onboard system that the residuals have changed (when a fault has occurred).

This section has been included to give only the most basics on change detectors; the more complete description is given in Lajic [15].

4.2. Change detectors. Sequential change detection algorithms, or sequential detectors, are the most common tools for detecting changes in a stochastic system. In principle, two groups of detectors can be distinguished, i.e. detectors for multiplicative faults and detectors for additive faults. The detectors for multiplicative faults are based on residual variance change detection [14], e.g. energy detectors, and, on the other hand, the detectors for additive faults are based on residual mean change detection, e.g. GLR (generalised likelihood ratio algorithm) [2] or CUSUM (cumulative sum algorithm) [25]. All these detectors use the Neyman-Pearson approach, cf. Kay [14]. According to the Neyman-Pearson approach the value of a likelihood ratio distinguishes between two hypotheses, i.e. the hypothesis that the fault is not present and the hypothesis that the fault is present. 

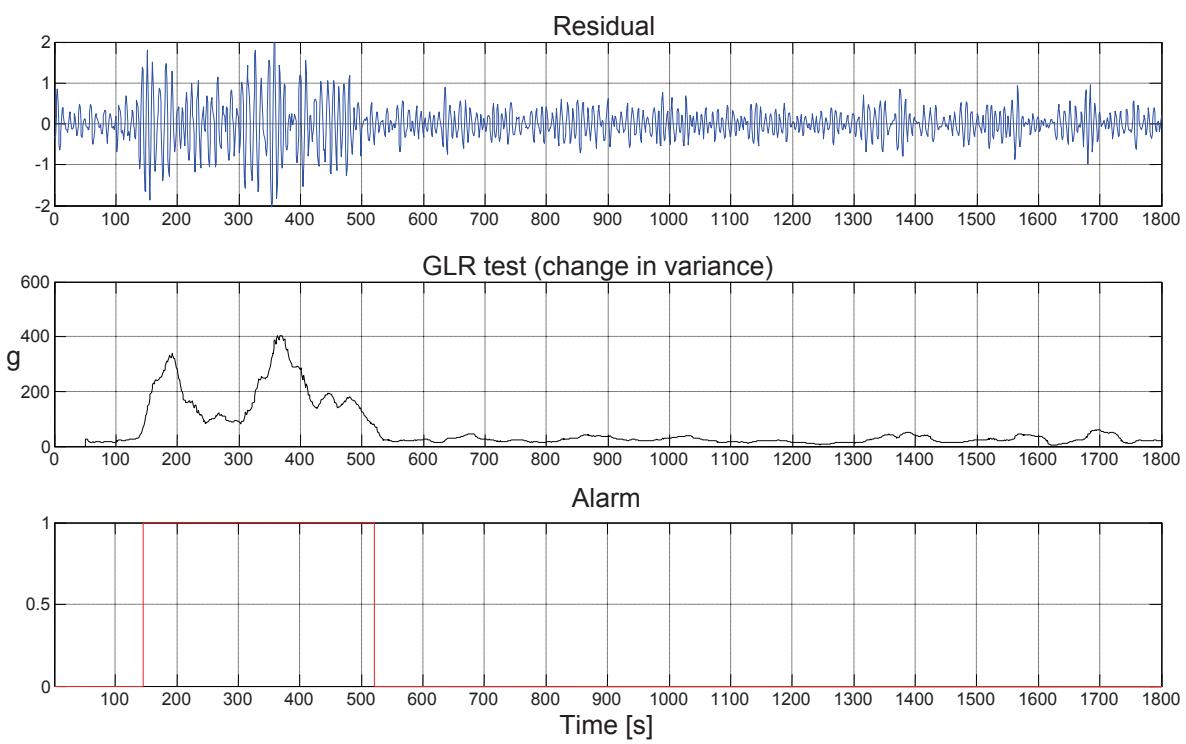

FigURE 9. Residual in the presence of a multiplicative fault between $100 \mathrm{~s}$ and 500

$s$ and GLR test (decision function and alarm). Lajic [15]

4.3. Decision function. Basically, change detectors are developed from assumptions about the probabilistic behaviour of the residual(s) and, mathematically speaking, the aim of a detector is to map a residual into a decision. In the Neyman-Pearson approach the mapping is controlled by a decision function together with a threshold value that must not be exceeded if the signal (residual) should be considered as non-faulty. In addition to the decision function itself, the determination of an appropriate threshold value becomes a central element for change detection. Means to decide on the threshold value have been discussed by, e.g., Galeazzi et al. [11]. An example of a decision function $g$ based on the GLR test is shown in Figure 9 for an arbitrary residual and an associated alarm function; further details can be found in [15].

\section{Fault detection And isolation}

5.1. Introduction. Onboard decision support systems rely fundamentally on receiving an estimate of the sea state, or the wave spectrum, as an input for the prediction of relevant/critical response values. One way to obtain an estimate of the - on-site - wave spectrum is to process a set of three measured ship responses by use of the wave buoy analogy, e.g. Nielsen [20, 21]. Typically, several responses are monitored on ships and it is therefore a matter of selecting a specific combination of these to be processed by the wave buoy analogy. Clearly, it is absolutely vital that faulty signals should be discarded from the procedure for sea state estimation if it is possible, if not, the fault 
TABLE 3. Main dimensions of the considered ship.

\begin{tabular}{lr}
\hline Length, $L_{\mathrm{pp}}$ & $275.0 \mathrm{~m}$ \\
Breadth, $B_{\mathrm{mld}}$ & $40.0 \mathrm{~m}$ \\
Draught, $T$ & $12.0 \mathrm{~m}$ \\
\hline
\end{tabular}

should be estimated. In this way, fault detection and isolation are remedies towards increased dependability and reliability of shipboard monitoring and decision support systems.

The present section serves to illustrate the derived models and the associated fault diagnosis techniques, when real data is analysed. Results will be given for a container ship, where focus is on full-scale motion measurements. For illustrative reasons, faults have been imposed artificially and, specifically, multiplicative faults are studied. It is noteworthy that other types of faults have been studied; results are given by Lajic [15].

5.2. Ship responses and imposed faults. The motion measurements have been obtained from a container ship with main dimensions as given by Table 3 . In the analysis four motion components are considered; the sway motion, the heave motion, the pitch motion and the vertical acceleration at the centre of gravity. Although the interest in this study does not directly concern sea state estimation, it should be noted that all of the four motion components can be used as candidates for the wave buoy analogy [24]. Time histories of the motion components are shown in Figure 10.

As examples of fault scenarios, faults will be created by introducing multiplicative faults $f_{i}=$ $k_{i} y_{i}\left(k_{i}=5, i=1, \ldots 4\right)$ to the individual motion components $i$. The faults are introduced during
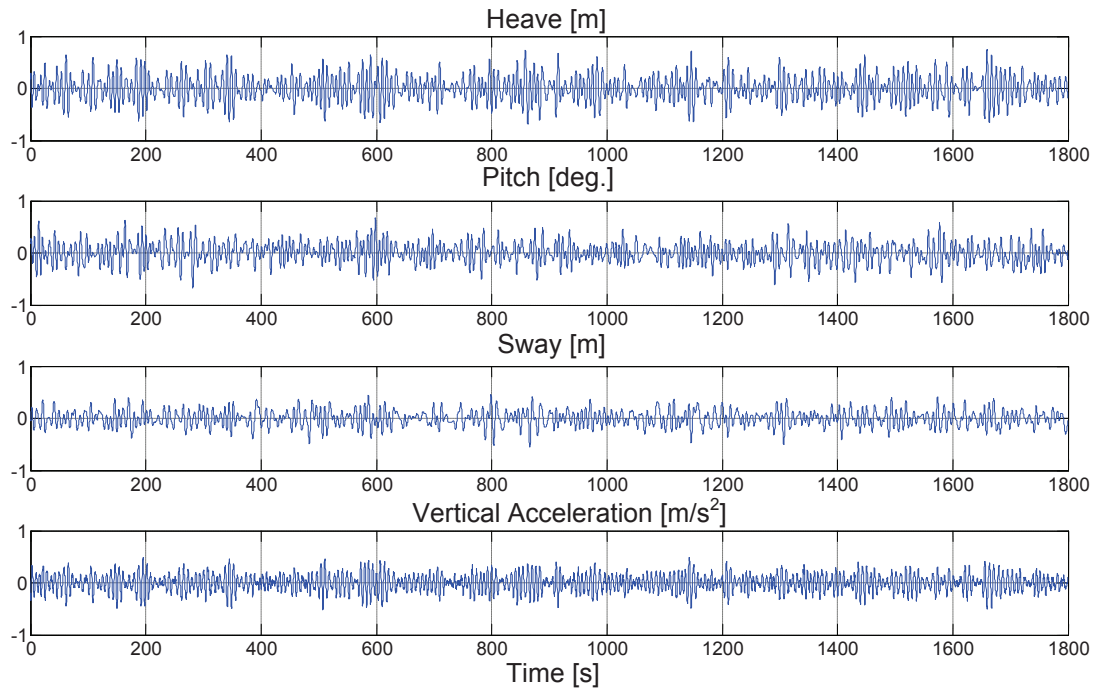

Figure 10. Measured time histories. 
TABLE 4. Fault signatures: $f_{1}$ is a fault on the heave sensor, $f_{2}$ is a fault on the pitch sensor, $f_{3}$ is a fault on the sway sensor, $f_{4}$ is a fault on the vertical acceleration sensor. The residuals $r_{1}-r_{6}$ are defined by Eqs. (36)-(41).

\begin{tabular}{|l|l|l|l|l|}
\hline$\nearrow$ & $f_{1}$ & $f_{2}$ & $f_{3}$ & $f_{4}$ \\
\hline$r_{1}$ & 1 & 1 & & \\
\hline$r_{2}$ & 1 & & 1 & \\
\hline$r_{3}$ & 1 & & & 1 \\
\hline$r_{4}$ & & 1 & 1 & \\
\hline$r_{5}$ & & 1 & & 1 \\
\hline$r_{6}$ & & & 1 & 1 \\
\hline & $i$ & $i$ & $i$ & $i$ \\
\hline
\end{tabular}

the time interval between $600 \mathrm{~s}$ and $1300 \mathrm{~s}$. In Figure 11 the ship responses in the presence of the multiplicative faults are shown. The value of the factor $k_{i}$ is for illustrative reasons chosen relatively large; the procedures have been tested and are well-working also for smaller values.

The fault signatures are shown in Table 4. All four column vectors are different from zero and have a unique signature, which means that all the faults are isolable. From the dependency matrix of the residuals $r_{1}, r_{2}$ and $r_{3}$ (cf. Figure 8), it is seen that violation of each constraint is isolable, except constraints $c_{1}$ and $m_{1}$. The fault $f_{1}$ is isolable but from a theoretical point of view, it is not
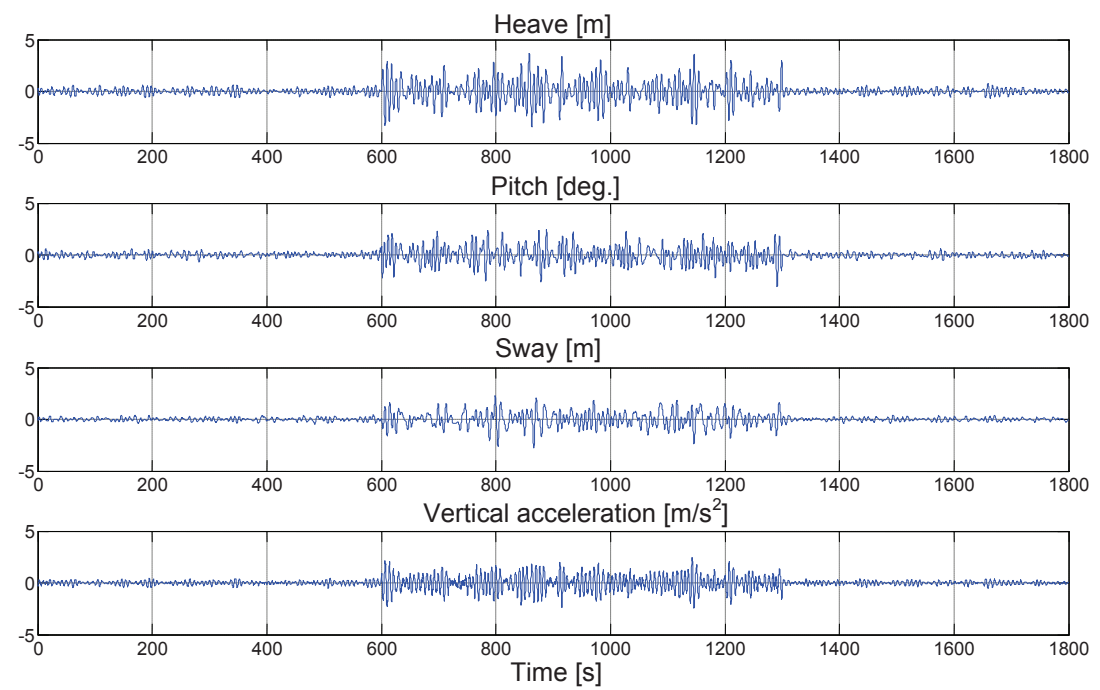

FIGURE 11. Ship responses with multiplicative faults between $600 \mathrm{~s}$ and $1300 \mathrm{~s}$. 
possible to distinguish if $c_{1}$ or $m_{1}$ is violated. From a practical point of view there is no difference; it is only important that all the faults can be isolated.

5.3. Residual evaluation. The direct calculation of the residuals, Eqs. (36)-(41), leads to dividing a response spectrum by the square of a corresponding transfer function. However, this division will, in general, cause numerical problems, since the transfer function(s) can have values equal to zero (or close to) for certain frequencies. Therefore, it will be more reliable to estimate the values of the residuals instead of calculating them directly. In the following, the residual evaluation is carried out by an approach that has some resemblance to the wave buoy analogy [20, 21], although the present procedure is developed to be more simple because the residual evaluation must be carried out continuously in real-time.

On the basis of Eqs. (36)-(41), the residuals are written in a general form as:

$$
r_{i}=\int\left[\frac{\left\{\mathfrak{F}\left(y_{j}\right)\right\}\left(\omega_{e}\right)}{\Phi_{j}^{2}\left(\omega_{e}\right)}\right] d \omega_{e}-\int\left[\frac{\left\{\mathfrak{F}\left(y_{k}\right)\right\}\left(\omega_{e}\right)}{\Phi_{k}^{2}\left(\omega_{e}\right)}\right] d \omega_{e} ; \quad j, k=1, \ldots 4 ; j \neq k ; \quad i=1, \ldots 6
$$

Moreover, the significant wave height is introduced from the calculation of the 0-th order spectral moment of the wave energy spectrum:

$$
\begin{aligned}
H_{s} & =4 \sqrt{m_{0}} \\
m_{0} & =\int_{0}^{\infty} S_{\zeta}\left(\omega_{e}\right) d \omega_{e}
\end{aligned}
$$

Thus, from the four constraints, $c_{1}, \ldots c_{4}$, and Eq. (43), the following expression can be obtained

$$
\int\left[\frac{\left\{\mathfrak{F}\left(y_{j}\right)\right\}\left(\omega_{e}\right)}{\Phi_{j}^{2}\left(\omega_{e}\right)}\right] d \omega_{e}=\left(\frac{H_{s, j}}{4}\right)^{2}
$$

where $H_{s, j}$ is the significant wave height that may be associated to the process which governs the signal $y_{j}$. Similarly, the signal $y_{k}$ can be used to establish the identical expression for the $k$-th process:

$$
\int\left[\frac{\left\{\mathfrak{F}\left(y_{k}\right)\right\}\left(\omega_{e}\right)}{\Phi_{k}^{2}\left(\omega_{e}\right)}\right] d \omega_{e}=\left(\frac{H_{s, k}}{4}\right)^{2}
$$

In this way, the problem of the residual calculation becomes a problem of estimating the significant wave height by use of different signals:

$$
r_{i}=\left(\frac{H_{s, j}}{4}\right)^{2}-\left(\frac{H_{s, k}}{4}\right)^{2} ; \quad j, k=1, \ldots 4 ; j \neq k ; \quad i=1, \ldots 6
$$

Consequently, the approach for fault detection and isolation becomes itself a matter of sea state estimation. However, to simplify and to avoid numerical problems, some elementary assumptions will be made. First of all, a (uni-modal) parameterised wave spectrum is introduced to describe 
the wave energy distribution as function of (wave) frequency $\omega$. Herein, the Pierson-Moskowitz spectrum is considered [13]:

$$
S_{P M}(\omega)=4 \pi^{3} H_{s}^{2} T_{z}\left(\omega T_{z}\right)^{-5} e^{-\pi^{3}\left(\omega T_{z} / 2\right)^{-4}}
$$

where $T_{z}$ is the zero-crossing period. Two other assumptions are imposed, so that (1) only longcrested waves are considered, and (2) the wave direction is taken to be identical to the wind direction. All together, the residual evaluation has been transformed into an optimisation problem in the variables $H_{s}$ and $T_{z}$. The cost function is established somewhat arbitrarily by comparing, frequency-wise, calculated and measured values of a given response spectrum and integrating. The cost function takes the form

$$
F_{i} \equiv \int_{0}^{\infty}\left[S_{P M}(\omega) \Phi_{i}^{2}(\omega)\left|\frac{d \omega}{d \omega_{e}}\right|-S_{i}\left(\omega_{e}\right)\right]^{2} d \omega_{e}
$$

where $S_{i}$ is the response spectrum obtained from the signal $y_{i}$. This means that an optimised set of parameters $\left(H_{s}, T_{z}\right)$ is determined from each of the considered sensor signals, and from this set the significant wave height is applied to Eq. (47).

5.4. Results and discussion. Multiplicative faults, $f_{i}=k_{i} y_{i}\left(k_{i}=5, i=1, \ldots 4\right)$, have been imposed to all sensor signals, and an example of the behaviour of one of the residuals formed by Eq. (47) is given in Figure 12. In the plots, the residual $r_{3}$ is shown in a non-faulty case (upper plot) and in the presence of faults on the heave (middle plot) and the acceleration (lower plot) sensors. The sensitivity of $r_{3}$ to a fault, being it on one or the other sensor, is clearly seen. The
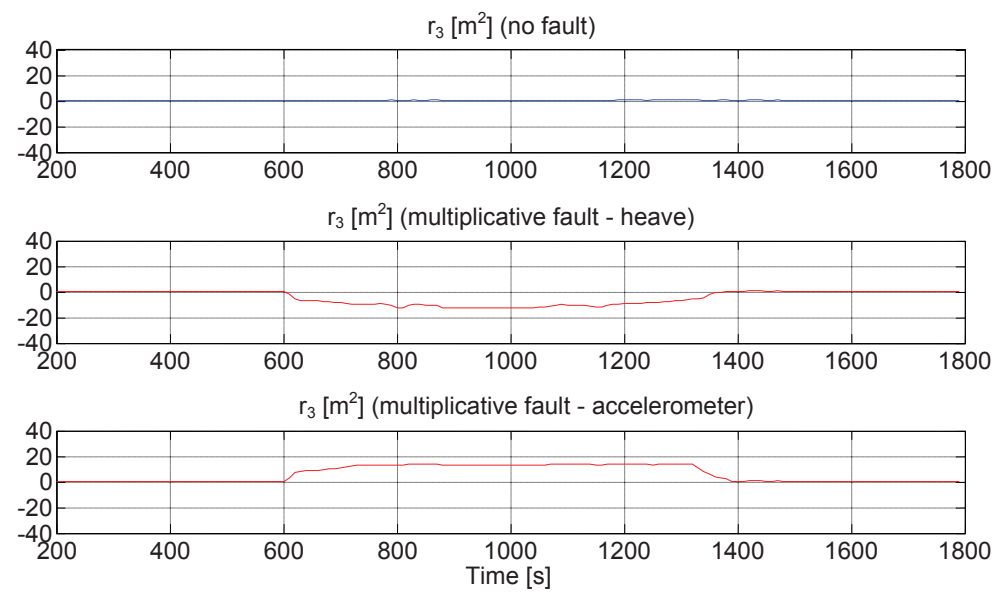

FIGURE 12. Residual $r_{3}$ in non-faulty and faulty cases. 
other residuals, $r_{1}, r_{2}, r_{4}, r_{5}, r_{6}$, behave very similar and, although not shown, the plots, made like those in Figure 12, exhibit an identical sensitivity to the imposed faults, cf. Lajic [15].

In the case of residual evaluation in the presence of a multiplicative fault, it is common to use detectors for change in the variance of the residual $[14,15]$. However, in the present work the residuals have been obtained by an optimisation procedure from the measured signals. Therefore, even in the case of a multiplicative fault, the presence of a fault will be reflected as a change in the mean value of the residual. For this reason the residuals are evaluated by a scalar GLR test [15]. The alarm diagram is constructed using a decision function and an appropriate threshold. The value of the threshold has been selected using an empirical method outlined by Galeazzi et al. [11]. In this approach the threshold value should be determined on the basis of healthy and faulty data. A computation of the decision function based on a set of healthy data makes it possible to determine the typical range of values of this function in the absence of fault, and to set the threshold in such a way that false alarms are avoided [8], which is absolutely fundamental for the end-users to have trust in any developed system. Details of the applied empirical method is found in Lajic $[15]$.

The results of the GLR test (decision function and alarm) for the residual $r_{1}$ are shown in Figure 13. It is easily seen that the system is in an alarm condition in the time interval between 600 seconds and 1300 seconds, which is the interval of the presence of the fault. In Figures 14-18 the results of the GLR test for the other residuals are given. It is seen that the GLR test is able to
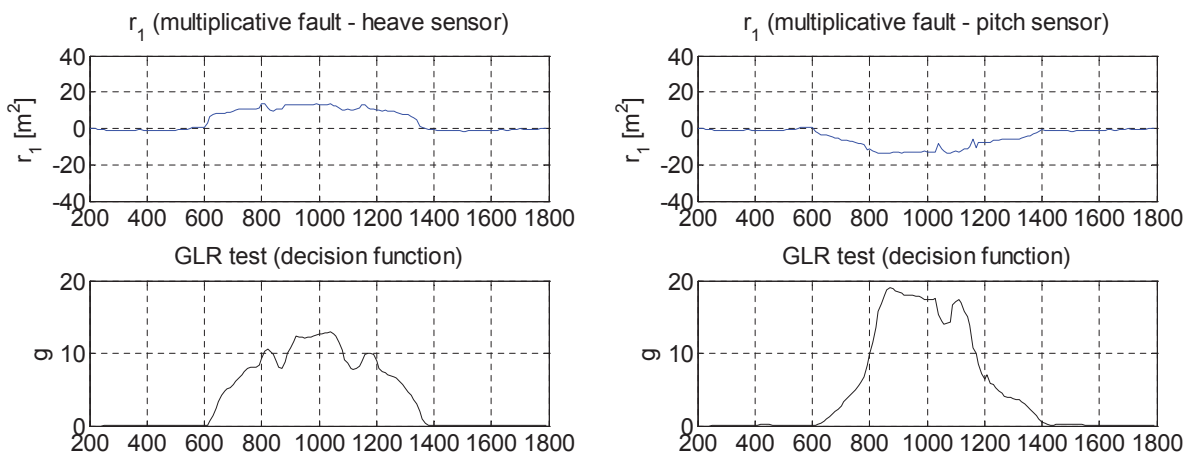

GLR test (alarm)
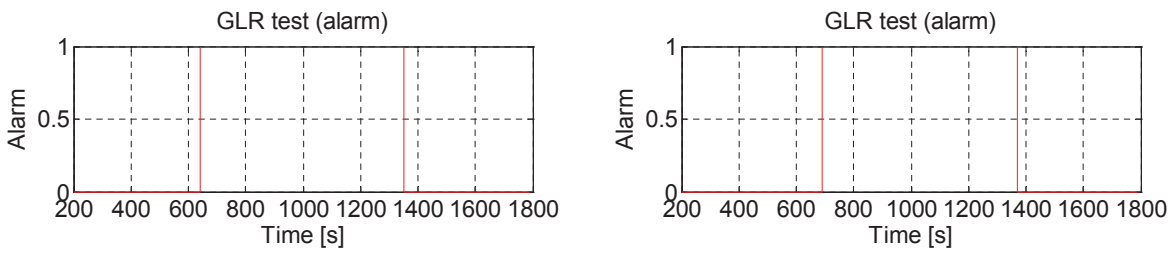

FIGURE 13. Residual $r_{1}$ in faulty cases for heave (left) and pitch (right). GLR test results - decision function and alarm. 
detect the presence of the faults in all the residuals. In general, it is observed that the GLR test turns the alarm on and off instantaneously, at the initiation of the fault and at the end, although a small delay in the detection is noticed by close inspection. However, in practice, it is typically a valid assumption to consider the sea state as changing slowly. Thus, the sea state is assumed to remain essentially constant for periods of the order of 20 to 30 minutes at least. Consequently, a small delay in the alarm is not critical for this kind of onboard systems.
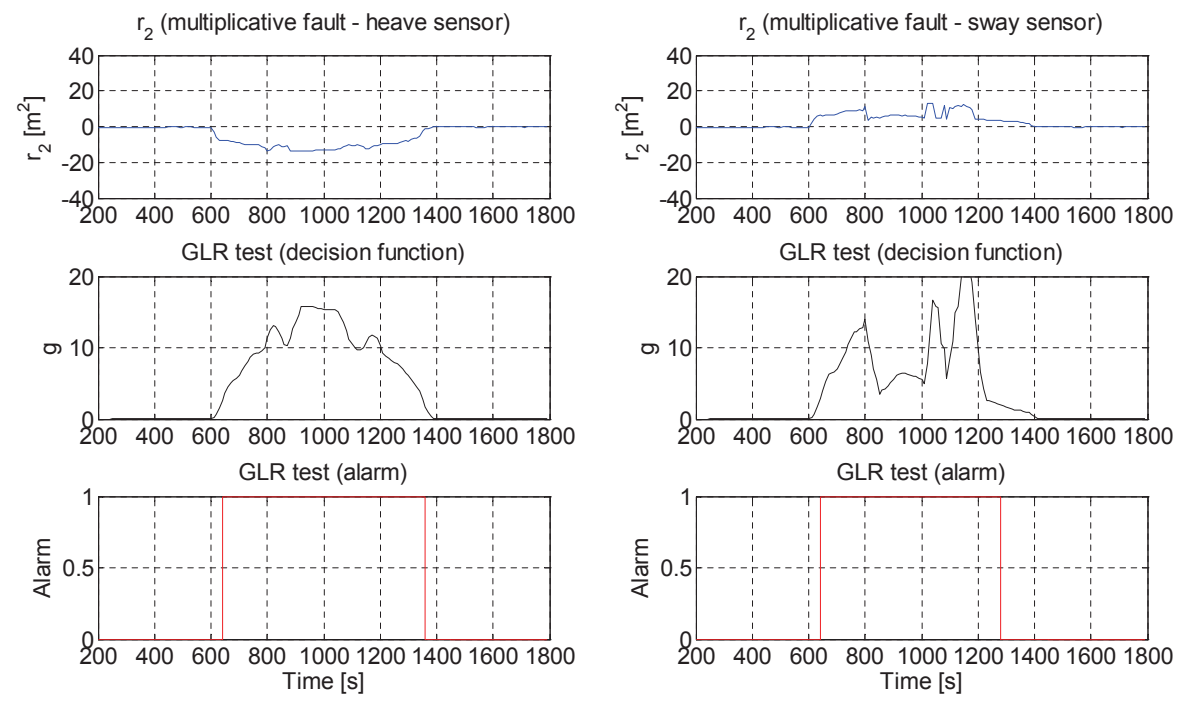

FIGURE 14. Residual $r_{2}$ in faulty cases for heave (left) and pitch (right). GLR test results - decision function and alarm.
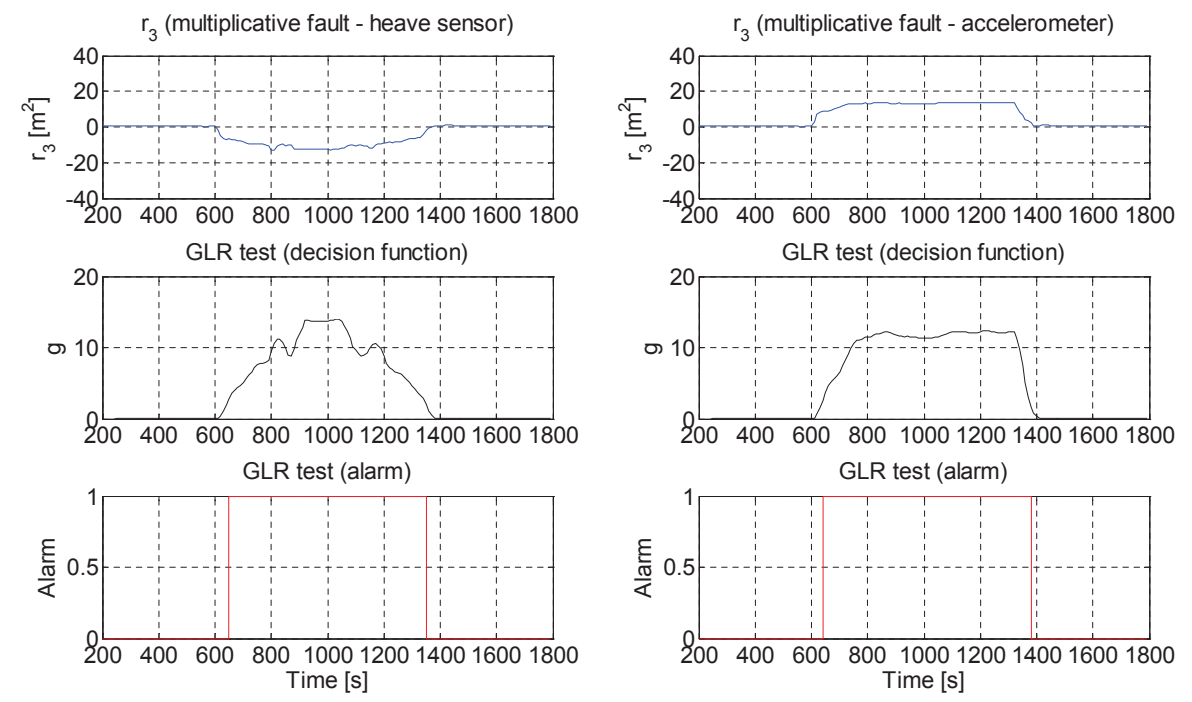

FIGURE 15. Residual $r_{3}$ in faulty cases for heave (left) and pitch (right). GLR test results - decision function and alarm. 

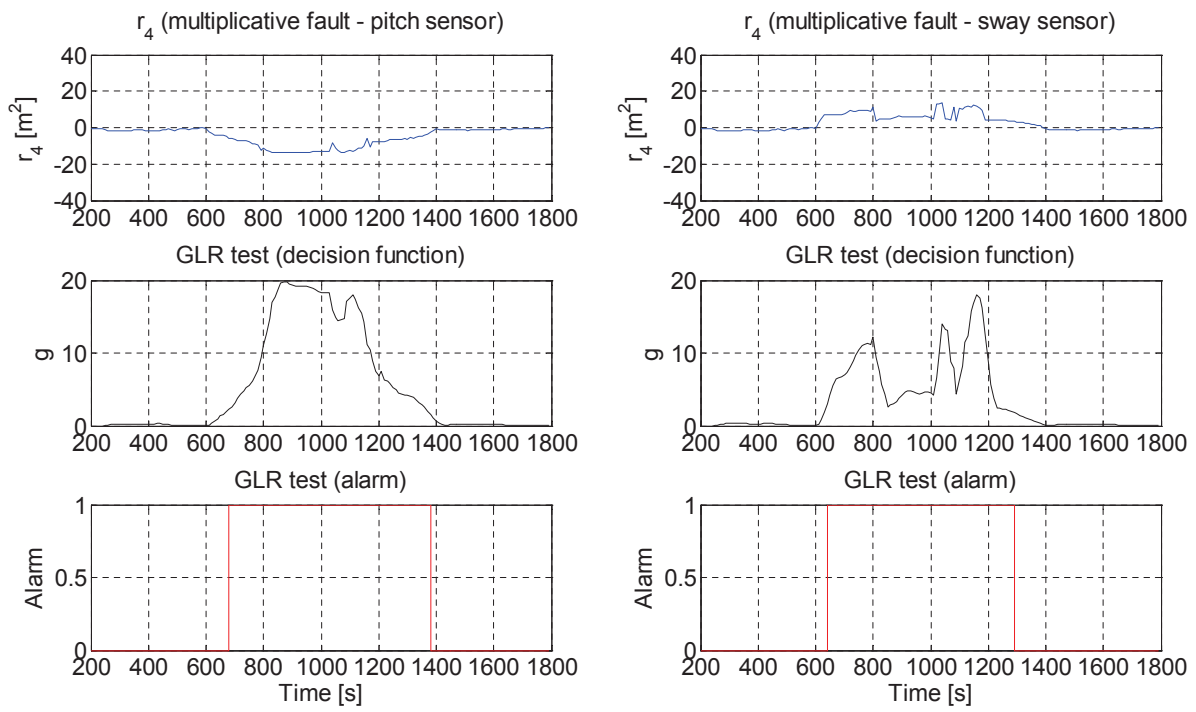

FIGURE 16. Residual $r_{4}$ in faulty cases for heave (left) and pitch (right). GLR test results - decision function and alarm.
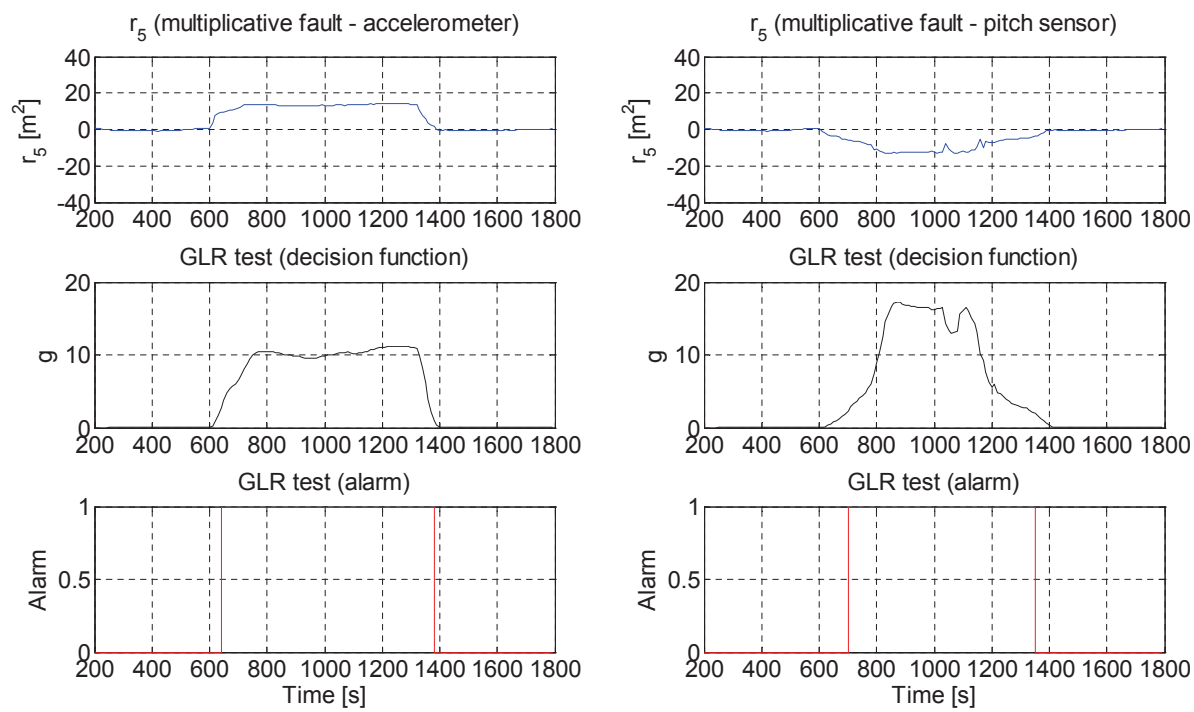

FigURE 17. Residual $r_{5}$ in faulty cases for heave (left) and pitch (right). GLR test results - decision function and alarm. 

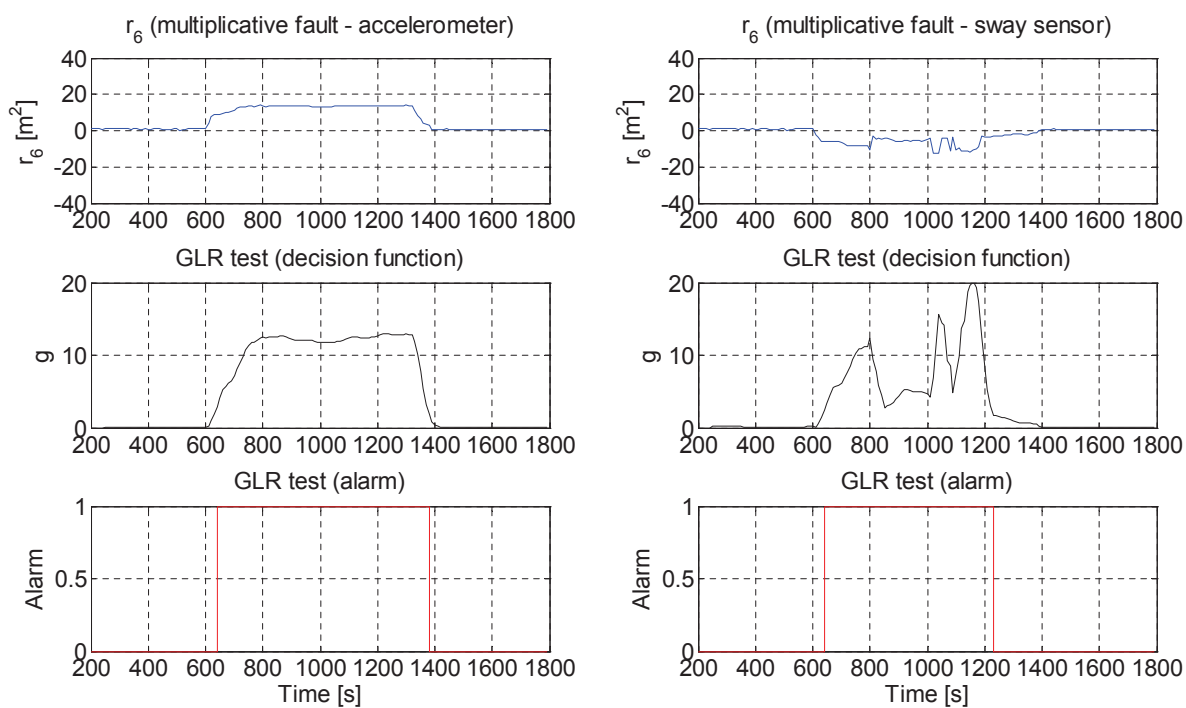

FIGURE 18. Residual $r_{6}$ in faulty cases for heave (left) and pitch (right). GLR test results - decision function and alarm.

\section{Summary AND CONCLUSIONS}

In the present work, ideas and approaches to increase the reliability and dependability of monitoring and decision support systems have been developed. In this context, matters of ship safety are the main concern for the studied systems. Specifically, fault diagnosis techniques have been considered for onboard systems aiming at operator guidance with respect to course and speed of the vessel. Decision support systems needs an estimate of the on-site sea state as a fundamental input. In case sea state estimation is conducted by the wave buoy analogy [20,21] the best solution is to use three different ship responses and usually several responses are available on today's commercial and naval vessels. However, faulty signals should be discarded from the procedure for sea state estimation if it is possible, if not the fault should be estimated.

In the article a specific and novel model for fault diagnosis have been discussed. The new numerical procedure is derived in the frequency domain and relies on linear spectral analysis. In this way, it is possible to define equations which correlate different ship responses through corresponding transfer functions. The model was tested using full-scale motion measurements from a container ship, where the data was analysed as a post-voyage process. The following points can be associated to the outcome of the test:

- Multiplicative faults were added artificially to the motion measurements and the sensitivity of the residuals to the sensor fault was investigated. 
- The residuals were based on wave height estimation and were evaluated by the GLR test.

- All the imposed faults were detected and isolated.

Fault detection and isolation are very important elements in the design of fault-tolerant decision support systems and this study has outlined remedies that can be applied for this purpose, when the ship responses are assumed to be linear in the wave excitation. In case of a non-linear model, Lajic [15] should be consulted and [15] also discusses the possibility to use Volterra theory for transforming a non-linear time domain model into a frequency domain model without linearisation.

6.1. Future work. In the future, the frequency domain model should be made even more robust. In particular, it would be of interest to consider the behaviour to faults imposed as (slow) drifts in signals, since this is a difficult but highly relevant problem. Moreover, it should be considered to relax on the crude assumptions imposed in the sea state estimation carried out as part of the residual evaluation. This includes, among others, the introduction of more realistic bi-modal wave spectra, and to consider scenarios where the wave direction is not necessarily equal to the wind direction.

\section{ACKNOWLEDGEMENT}

The authors would like to express their sincere thanks to Professor Mogens Blanke for many valuable comments.

\section{REFERENCES}

1. M. Aschehoug, Scientific Paper on The Sea State Estimation Methodology, Tech. report, SIREHNA, France, 2003, Paper prepared in the HullMon+ project.

2. M. Basseville and I. Nikiforov, Detection of Abrupt Changes: Theory and Application, Prentice-Hall, 1993.

3. M. Blanke, Enhanced Maritime Safety Through Diagnosis and Fault Tolerant Control, Proc. 5th IFAC Conference, CAMS'2001 (Glasgow, UK), 2001, Invited pleanary.

4. __ Fault-tolerant and Diagnostic Methods for Navigation, Proc. 9th International Conference on Marine Engineering Systems, ICMES'2003 (Helsinki, Finland), 2003.

5. __ Diagnosis and Fault-tolerant Control for Ship Station Keeping, Symposium on Intelligent Control and 13th Mediterranean Conference on Control and Automation (Limassol, Cyprus), 2005.

6. _ Fault-tolerant Sensor Fusion for Marine Navigation, Proc. 7th IFAC Conference on Manoeuvring and Control of Marine Craft (Lisbon, Portugal), 2006.

7. M. Blanke, R. Izadi-Zamanabadi, and T.F. Lootsma, Fault Monitoring and Re-configurable Control for a Ship Propulsion Plant, International Journal of Adaptive Control and Signal Processing 12 (1998), 253-263.

8. M. Blanke, M. Kinnaert, J. Lunze, and M. Starosweicki, Diagnosis and Fault-tolerant Control, Springer, 2006. 
9. DNV, User Manual: Wasim, 2005, (Technical documentation by DNV.).

10. R. Galeazzi, M. Blanke, and N.K. Poulsen, Detection of Parametric Roll Resonance on Ships from Indication of Nonlinear Energy Flow, Proc. 7th IFAC Symposium on Fault Detection, Supervision and Safety of Technical Processes (Barcelona, Spain), 2009.

11. __ Parametric Roll Resonance Detection using Phase Correlation and Log-likelihood Testing Techniques, Proc. 8th IFAC International Conference on Manoeuvring and Control of Marine Craft, MCMC'2009 (Guaruja, Brazil), 2009.

12. T. Iseki and K. Ohtsu, Bayesian estimation of directional wave spectra based on ship motions, Control Engineering Practice 8 (2000), 215-219.

13. J.J. Jensen, Load and Global Response of Ships, Elsevier Ocean Engineering Book Series, vol. 4, Elsevier, 2001.

14. S.M. Kay, Fundamentals of statistical signal processing: Detection theory. Vol. II, Prentice-Hall PTR, 1998.

15. Z. Lajic, Fault-tolerant Onboard Monitoring and Decision Support Systems, Ph.D. thesis, Department of Mechanical Engineering, Technical University of Denmark, December 2010.

16. Z. Lajic, M. Blanke, and U.D. Nielsen, Fault Isolation for Shipboard Decision Support, Proc. 7th IFAC Symposium on Intelligent Autonomous Vehicles (Lecce, Italy), IFAC, 2010.

17. Z. Lajic and U.D. Nielsen, Fault Detection for Shipboard Monitoring and Decision Support Systems, Proc. of OMAE'09 (Honolulu, HI, USA), ASME, 2009.

18. Z. Lajic, U.D. Nielsen, and M. Blanke, Fault Isolation and Quality Assessment for Shipboard Monitoring, Proc. 29th OMAE (Shanghai, China), ASME, 2010.

19. J.G. Miller, Living Systems, University Press of Colorado, 1995.

20. U.D. Nielsen, Estimations of on-site directional wave spectra from measured ship responses, Marine Structures 19 (2006), 33-69.

21. Introducing two hyperparameters in Bayesian estimation of wave spectra, Probabilistic Engineering Mechanics 23 (2008), 84-94.

22. U.D. Nielsen and J.J. Jensen, A novel approach for navigational guidance of ships using onboard monitoring systems, Ocean Engineering 38 (2011), 444-455.

23. U.D. Nielsen, J.J. Jensen, P.T. Pedersen, and Y. Ito, Onboard monitoring of fatigue damage rates in the hull girder, Marine Structures 24 (2011), 182-206.

24. U.D. Nielsen and D.C. Stredulinksy, Sea state estimation from an advancing ship - A comparative study using sea trial data, Applied Ocean Research 34 (2012), 33-44.

25. E.S. Page, Continuous Inspection Schemes, Biometrika 41 (1954), 100-115.

26. R. Pascoal and C. Guedes Soares, Kalman filtering of vessel motions for ocean wave directional spectrum estimation, Ocean Engineering 36 (2009), 477-488.

27. R. Pascoal, C. Guedes Soares, and A.J. Sørensen, Ocean Wave Spectral Estimation Using Vessel Wave Frequency Motions, Journal of Offshore Mechanics and Arctic Engineering 129 (2007), 90-96.

28. M. St.Denis and W.J. Pierson, On the Motion of Ships in Confused Seas, Trans. of SNAME 61 (1953), 280-332.

29. E.A. Tannuri, J.V. Sparano, A.N. Simos, and J.J. Da Cruz, Estimating directional wave spectrum based on stationary ship motion measurements, Applied Ocean Research 25 (2003), 243-261. 
30. A. Tiano, Z. Lajic, and M. Carreras, Adaptive Control of Underwater Vehicles, Proc. 7th IFAC International Conference on Manoeuvring and Control of Marine Craft, MCMC'2006 (Lisbon, Portugal), 2006.

31. N.E.S. Wu, S. Thavamani, Y.M. Zhang, and M. Blanke, Sensor Fault Masking of a Ship Propulsion System, Control Engineering Practice 14 (2006), 1337-1345. 